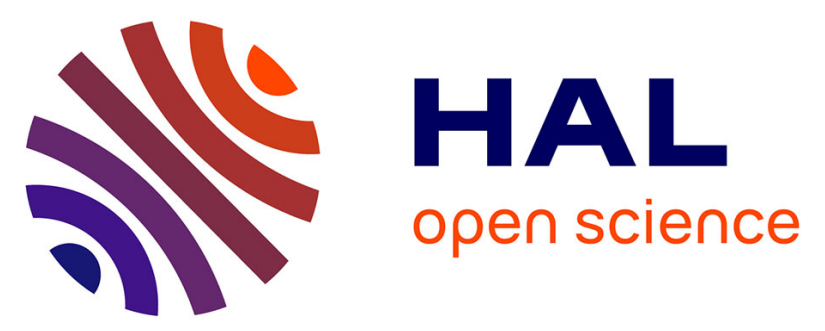

\title{
An original method to determine complex refractive index of liquids by spectroscopic ellipsometry and illustrated applications
}

\author{
M. Stchakovsky, Y. Battie, A. En Naciri
}

\section{- To cite this version:}

M. Stchakovsky, Y. Battie, A. En Naciri. An original method to determine complex refractive index of liquids by spectroscopic ellipsometry and illustrated applications. Applied Surface Science, 2017, 421 (Part B), pp.802-806. 10.1016/j.apsusc.2016.12.001 . hal-02899469

\section{HAL Id: hal-02899469 \\ https://hal.univ-lorraine.fr/hal-02899469}

Submitted on 28 Jan 2022

HAL is a multi-disciplinary open access archive for the deposit and dissemination of scientific research documents, whether they are published or not. The documents may come from teaching and research institutions in France or abroad, or from public or private research centers.
L'archive ouverte pluridisciplinaire HAL, est destinée au dépôt et à la diffusion de documents scientifiques de niveau recherche, publiés ou non, émanant des établissements d'enseignement et de recherche français ou étrangers, des laboratoires publics ou privés.

\section{(ㅇ)(1) $\$$}

Distributed under a Creative Commons Attribution - NonCommercial - NoDerivatives 44.0 


\title{
An Original Method to Determine Complex Refractive Index of Liquids by Spectroscopic Ellipsometry and Illustrated Applications
}

\author{
M. Stchakovsky ${ }^{1 *}$, Y. Battie ${ }^{2}$, A. En Naciri²
}

1. Horiba Scientific, Avenue de la Vauve, Passage Jobin Yvon, 91120 Palaiseau, France

2. LCP-A2CM, Institut Jean Barriol, Université de Lorraine, 1 Bld Arago, 57070 Metz, France

*corresponding author, michel.stchakovsky@horiba.com

\begin{abstract}
We present a method to characterize optical properties of liquids by spectroscopic ellipsometry. The experiments use a specific liquid cell that avoids disturbance of waves at air-liquid interface and allows the determination of the real and the imaginary part of the refractive index, with a sensitivity of the latter below $10^{-4}$. The method is illustrated by results obtained with a spectroscopic phase modulation ellipsometer on several liquids such as deionised water, microscope oil and protein solution. Comparisons of the method with standard techniques are given.
\end{abstract}

Keywords: Ellipsometry; Liquid, Optical properties; Water; Immersion liquid; BSA concentration

\section{Introduction}

Ellipsometry has become a reference technique that provides optical properties which allow one to investigate or qualify material intrinsic properties. A survey of the scientific literature and industrial applications reveals that spectroscopic ellipsometry is applied mostly to characterize solids and generally thin film samples with a more or less complex structure [1]. Ellipsometry has also been applied to study solids in liquid ambients [2] and liquid-liquid or air-liquid interfaces [3-6] but to our knowledge, there has not been an application of ellipsometry to efficiently characterize real and imaginary parts of the complex refractive index of the actual liquids. There are several reasons for this, the most realistic one being 
that for liquids, transmission spectroscopy remains the reference technique, allowing reasonably good determination of the extinction coefficient. However transmission spectroscopy does not inform independently on the real part of the refractive index, for which Kramers-Kronig methods are required. On the other hand, ellipsometry is the only technique that gives access to both the real and the imaginary part of the optical indices of materials $(N=n+i k)$ from measurement of the ellipsometric angles $\psi$ and $\Delta$ at incidence angle $\Phi$ in air, simply by applying the two phases (bulk-air) semi-infinite model :

$$
\rho=\tan \psi e^{i \Delta} \quad \text { and } N^{2}=\sin ^{2} \Phi\left[1+\tan ^{2} \Phi \frac{(1-\rho)^{2}}{(1+\rho)^{2}}\right]
$$

\section{Limitations of the bulk modelling approach}

A difficulty met when measuring bulk materials by ellipsometry is the inevitable influence of overlayers, even at the nanometer scale because of the sensitivity of the technique. For bulk solids, this overlayer is often a combination of both roughness and contamination or oxidation. Its presence rules out the possibility to apply the semi-infinite direct inversion equation (1). For liquids, similar difficulty takes place with the overlayer being due to capillary waves. We illustrate in Figure 1 what may be the most critical influence of these surface defects to ellipsometric analysis : it is impossible to distinguish between two systems such as a transparent liquid of pure real index covered by $1.2 \mathrm{~nm}$ overlayer (Fig. 1a) from a slightly absorbing liquid of complex index covered by $1 \mathrm{~nm}$ overlayer (Fig. 1b) because both systems generate nearly identical ellipsometric response (Fig 1c) even at different angles of incidence. Inputs for these simulations are :

- $\mathrm{n}$ vary between 1.33 to 1.44 and $\mathrm{k}$ vary between $3.10-4$ to $2.10-3$ from near infrared to ultraviolet following an harmonic oscillator dispersion formula

- angles of incidence vary every $0.5^{\circ}$ between $52.5^{\circ}$ and $54.5^{\circ}$, on both sides of Brewster angle of the liquid, in order to improve the sensitivity of the ellipsometric data to the overlayer. 

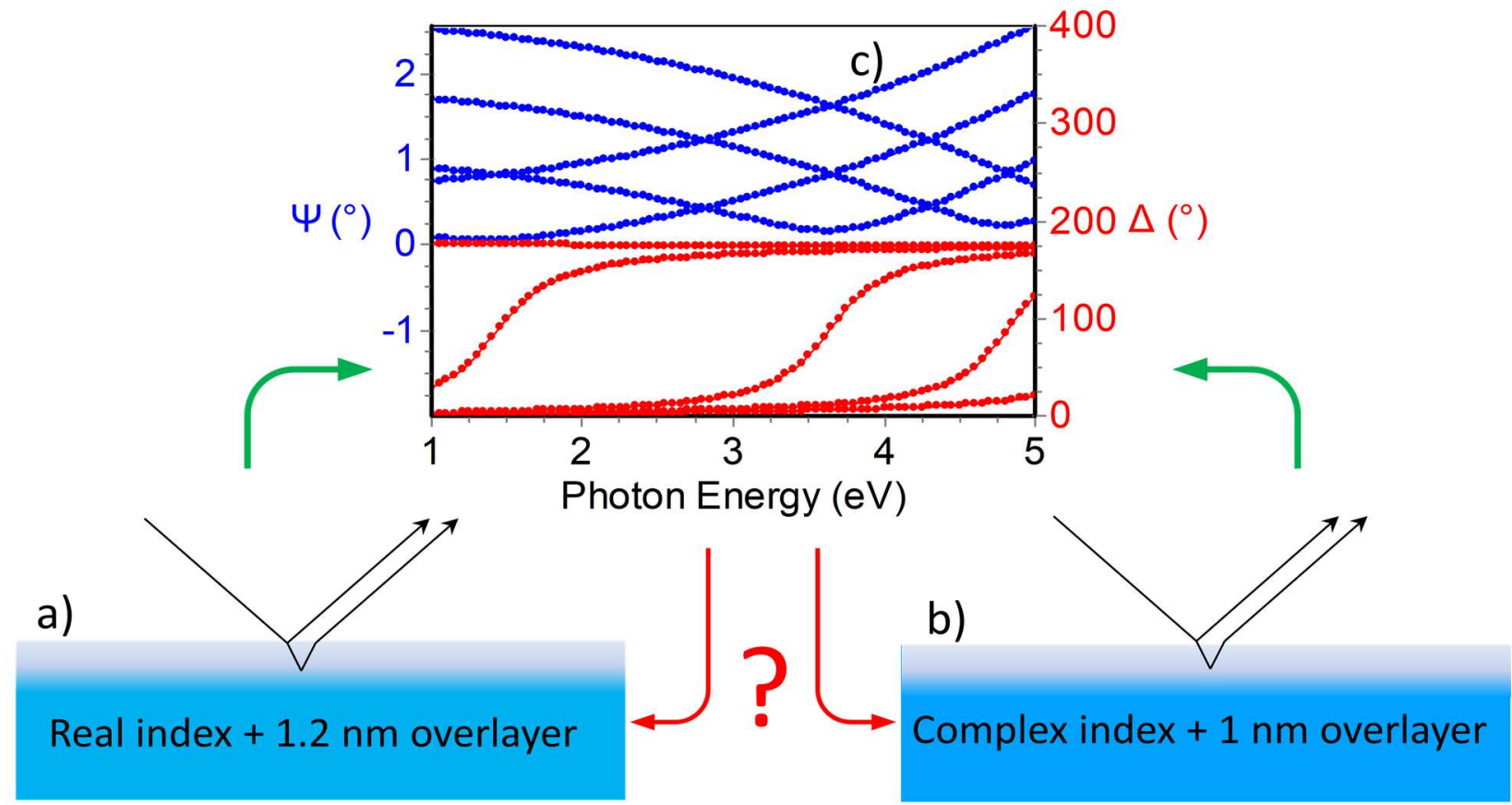

Figure 1 Illustration for ambiguous ellipsometric inverse problem (red arrows). Two different models a and $b$ having similar ellipsometric signature at angles of incidence varying between $52.5^{\circ}$ and $54.5^{\circ}(c)$

This correlation can explain the lack of studies on liquids by standard reflexion ellipsometry. It also illustrates the general limitation of the technique to detect small absorptions of bulk phases. The reason why this theoretical example is given is because the technique described in the next section takes advantage of a new principle to overcome the difficulties encountered with liquid overlayers or weak absorptions.

\section{Experimental principle and background for modeling}

We propose an ellipsometric setup to characterize the liquids that combines several specific aspects which are summarized in Fig 2 . 


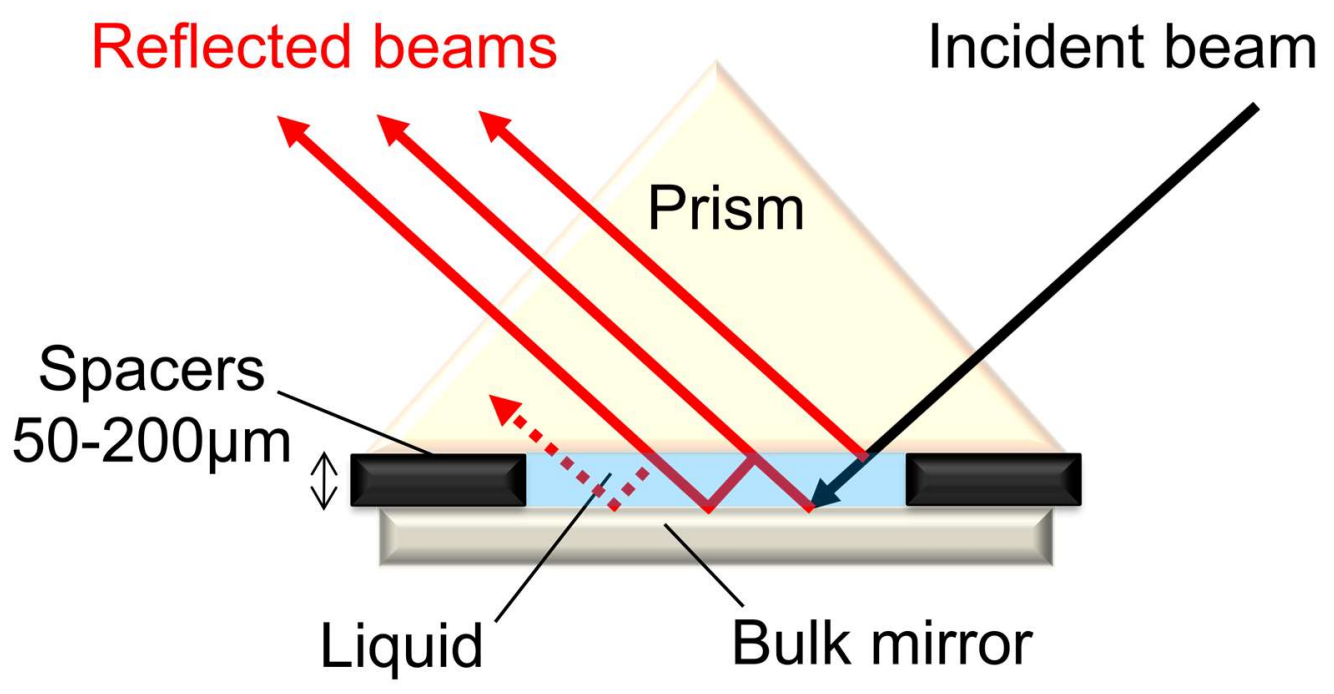

Figure 2 Principle of the method. Multiple reflected beams become negligible after the third one

The liquid under study is sandwiched between the base of an isosceles prism and a bulk mirror. This ensures the absence of surface waves at both interfaces of the liquid. The thickness of the liquid under study is mechanically fixed by spacers with thicknesses in the range of 50 to $200 \mu \mathrm{m}$. The incident ellipsometric beam enters the prism under normal incidence and the angle of the prism and its material are so that light is partially reflected and transmitted at the prism-liquid interface. This method should not be confused with the total reflection technique [5]. Finally, light is reflected at the liquid-mirror interface. A regime of multiple reflections takes place into the liquid layer when it is sufficiently transparent. The thickness of the liquid between spacers makes the optical path of multiple reflections at oblique incidence in the range of $200 \mu \mathrm{m}$ and more. Such a transmitted path of light through the liquid enables a very significant improvement of sensitivity to the small absorption of the liquid compared to the one obtained during the bulk studies.

In such a regime of multiple reflections into the liquid, given the typical range of thicknesses, there are no interferences as the path difference between multiple emerging beams is larger than the coherency length of the optical setup, which is given by the spectral resolution of the detection system, typically in the range of $4 \mathrm{~nm}$. Thus, regarding thin film optics, the modeling for such experiments consists of the incoherent summation of multiple reflected beams, adding their contributions in terms of intensities. The estimation of the related intensities between each beam shows that one can neglect the contributions of beams after the third one as illustrated in Fig.2. The modeling process comparing 
measured data and calculated one relies on the approach developed in ref [7]. Measured data are $\mathrm{m}_{33}$ and $\mathrm{m}_{34}, 2$ elements of the Mueller matrix of the system, often mentioned as variable Is and Ic. With brackets <> denoting products averaged over spectral resolution, the ellipsometric parameters Is and Ic have the following form :

$$
\mathrm{m}_{33}=\mathrm{Ic}=\frac{\left.2 \operatorname{Re}<r_{p} r_{s}^{*}\right\rangle}{\left\langle r_{p} r_{p}^{*}\right\rangle+\left\langle r_{s} r_{s}^{*}\right\rangle} \text { and } \mathrm{m}_{34}=\mathrm{Is}=\frac{\left.2 I m<r_{p} r_{s}^{*}\right\rangle}{\left.\left\langle r_{p} r_{p}^{*}\right\rangle+<r_{s} r_{s}^{*}\right\rangle}
$$

Calculated data come from the geometric serie summation of partial beam decomposition. When neglecting any overlayer surrounding the liquid, they take the form :

$$
<r_{x} r_{y}^{*}>=r_{01 x} r_{01 y}^{*}+\frac{t_{01 x} t_{01 y}^{*} t_{10 x} t_{10 y}^{*} r_{12 x} r_{12 y}^{*} \mathrm{e}^{-4 \operatorname{Im}(\beta)}}{1-r_{10 x} r_{10}^{*} r_{12 x} r_{12 y}^{*} \mathrm{e}^{-4 \operatorname{Im}(\beta)}}
$$

where $r$ (resp. t) stands for Fresnel reflection (resp. transmission) coefficient and the index $x$ or $y$ for either $p$ or $s$ polarization mode. The asterisk stands for complex conjugate, the indices 0, 1, 2 denote interfaces between media, respectively, prism, liquid and mirror. The film phase thickness $\beta$ depends on the thickness of the liquid $d$ and the wavelength $\lambda$ :

$$
\beta=2 \frac{\mathrm{d}}{\lambda}\left(\mathrm{n}_{1}^{2}-\mathrm{n}_{0}^{2} \sin ^{2} \Phi\right)^{1 / 2}
$$

A special device holding the prism, the liquid, the spacer and the mirror was developed in order to achieve the work shown here. The prism is made of fused silica with an isosceles angle smaller than critical angle, typically $55^{\circ}$. The cell is made of PVC and the bulk mirrors in the present studies are bare silicon wafers. Spacers are electro polished Ni blades with 3 sets of thicknesses between 50 and $200 \mu \mathrm{m}$, certified within $1 \%$. A channel in the PVC cell allows an easy liquid insertion with standard pipettes.

Experiments were performed over the range of 190 to $2100 \mathrm{~nm}$ on a UVISEL type spectroscopic phase modulation ellipsometer. This type of device fits the requested optical performances of the precise multiple beam collection thanks to its large aperture of collecting optics and mostly the use of fixed components as polarizing elements.

In future work, the choice of other angles of prism cuts and material can be investigated. This, together with the selection of a different bottom mirror, may lead to optimized sensitivity depending on the liquid under study. In the UV-NIR range, silica prisms have a 
strong advantage because they present the widest transparency range. However, for liquids with $\mathrm{n} \approx 1.46$, results may be different because the reflection at the prism/liquid interface would nearly vanish, so in this case and a high index glass prism will be more appropriate. In order to complete the optimal sensitivity studies, a different bottom mirror from bare silicon should be investigated as well. In liquids we have studied, reflectivity of such mirror changes approximately from $20 \%$ to $60 \%$ with wavelength without affecting significantly our results. Materials like Al could be used to enhance reflected intensity and reduce chromatic effects, however its stability in aqueous solution might generate other undesired phenomena. Indeed, overlayer on the mirror has an influence that has been taken into account. With silicon mirror, this interface was determined by measurements of bare wafer in water, water being the ambient material, performed prior to our experiments in liquids. From this preliminary study, in modeling illustrated in the next section, the bottom mirror includes a $4 \mathrm{~nm}$ layer of silicon oxide. On the other hand, from the opposite side of the liquid film, it has been observed and confirmed by sensitivity calculations that the influence of an interface between the prism and the liquid is negligible.

\section{Illustrated results}

\subsection{Water}

The validation of the proposed method was established by comparing our experiments on deionized water with the abundant literature dealing with this subject [8-12]. Figure 3 illustrates the comparison between the ellipsometric room temperature measurements (dots) and the model (solid line). 

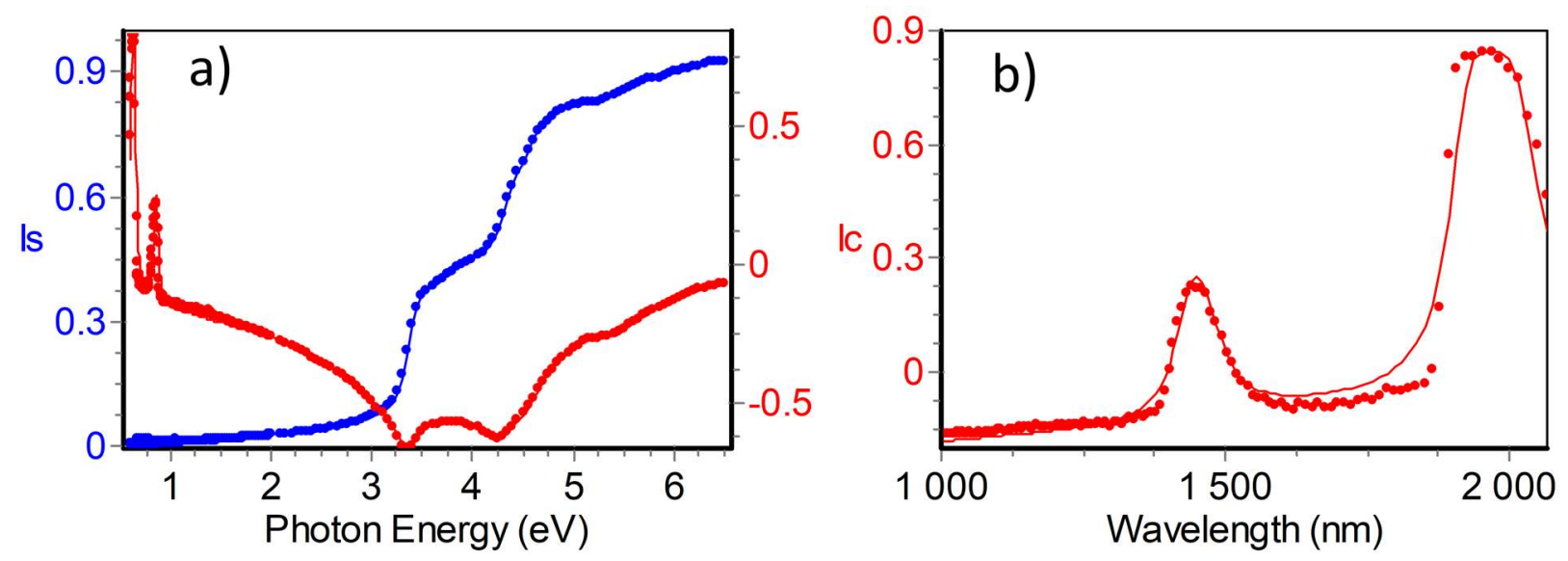

Figure 3 a) Comparison between ellipsometric measurements on water (dots) and calculated data (solid line). Is $=\sin 2 \psi \sin \Delta$; Ic $=\sin 2 \psi \cos \Delta$. b) magnification of Ic in near infrared range where water absorption modes are significant

This excellent fit is obtained by using a 4xTauc-Lorentz dispersion formula [13] in order to describe the optical properties of water. The corresponding graphs are given in Figures $4 a$ and $4 \mathrm{~b}$. These optical properties match the room temperature values from literature, in the spectral range of availability, within 0.005 on the real part of the index $\mathrm{n}$ and better than 0.001 on the imaginary part of the index $\mathrm{k}$. This is the order of magnitude of accuracy we will consider from now on with our technique. One must notice that discrepancies among literature values themselves reach more than 0.01 on $\mathrm{n}$, for example at $300 \mathrm{~nm}$ between ref [8] and [11].
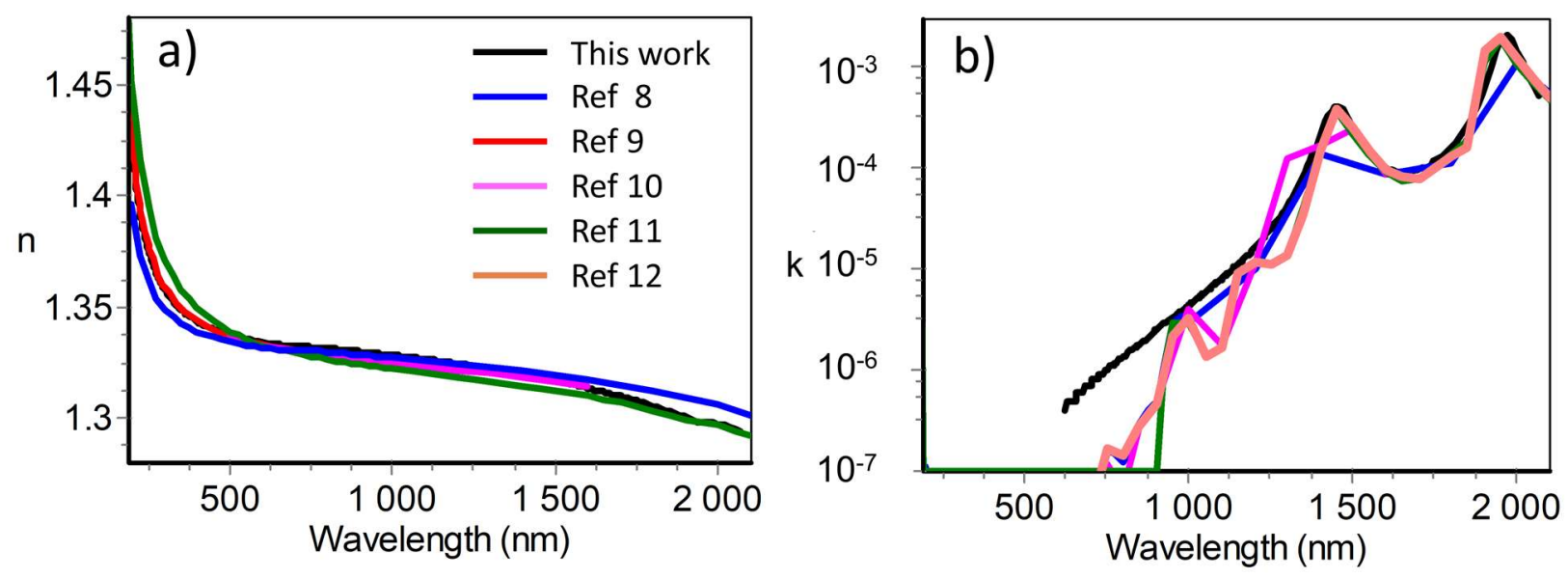

Figure 4 Room temperature optical properties of water obtained in this work and published in the literature. a) Real part and b) Imaginary part 
Figure $4 \mathrm{~b}$ shows that we did not determine extinction coefficient below $600 \mathrm{~nm}$, where its value becomes smaller than $10^{-6}$ the limit of sensitivity on $\mathrm{k}$ obtained by the method. It comes from loss by transmission through thickness of liquid crossed by light in the cell. Figure 5 illustrates the point. When light is transmitted through $200 \mu \mathrm{m}$, no significant losses take place and it becomes impossible to estimate $\mathrm{k}$ below $10^{-6}$ at any wavelength or even below $10^{-4}$ in NIR.

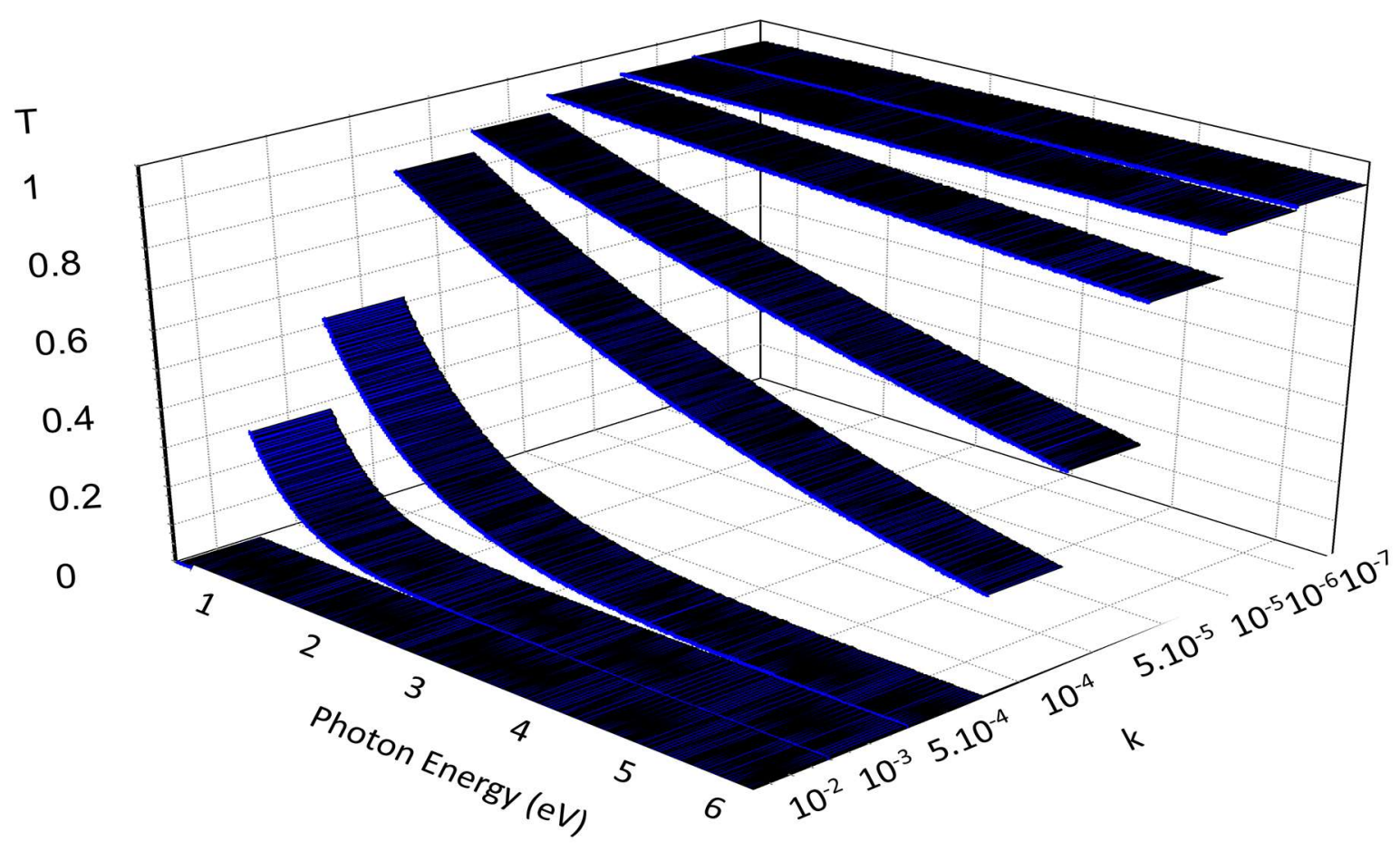

Figure 5 Transmission of light through $200 \mu \mathrm{m}$ of material according to extinction coefficient $k$ and energy in $\mathrm{eV}$. Transmission is nearly $100 \%$ for $\mathrm{k}<10^{-6}$.

\subsection{Ethanol and immersion liquids}

The technique was applied to extract optical properties of ethanol. As illustrated in Figure 6 a good agreement between the real part of refractive index calculated with a $2 x$ TaucLorentz formula and the tabulated values of Giessen [10] is obtained. The precise determination of extinction coefficient in NIR was not possible because so far it is below sensitivity limits. Still, figure $6 \mathrm{~b}$ shows good qualitative agreement between the published values of $k$ and experimental data for Ic. One can notice that these similarities between $k$ 
and Ic were already observed on Fig3b and Fig4b. Although claimed impossible and illustrated on Fig5 above, looking for improved optimal dispersion law or increasing spacer thickness and consequently optical path inside the liquid in order to fit these experiments and determine $\mathrm{k}$ below $10^{-4}$ is under investigation.
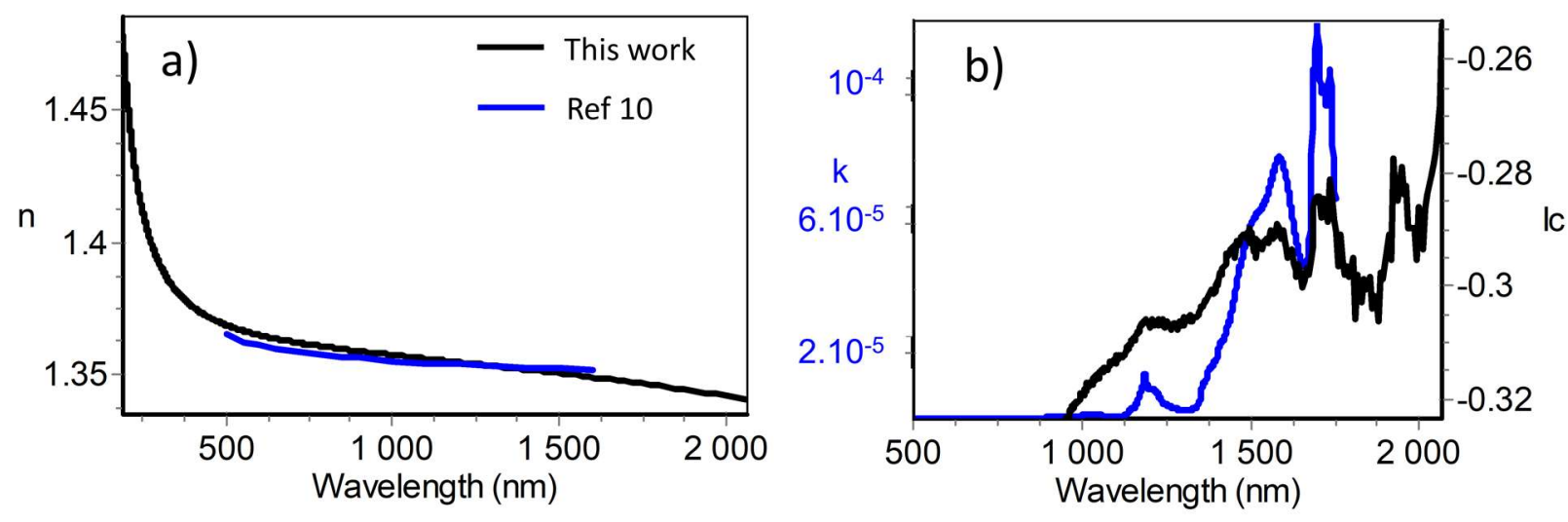

Figure 6 Experiments on ethanol. a) Comparison between the real part of refractive index obtained by ellipsometry and the one given in reference [10]. b) Qualitative comparison between the measured ellipsometric parameter Ic and the published extinction coefficient.

Two transparent fluorinated liquids for application in microscopy were analyzed and their optical indices obtained with a transparent harmonic oscillator law are illustrated in Figure 7.

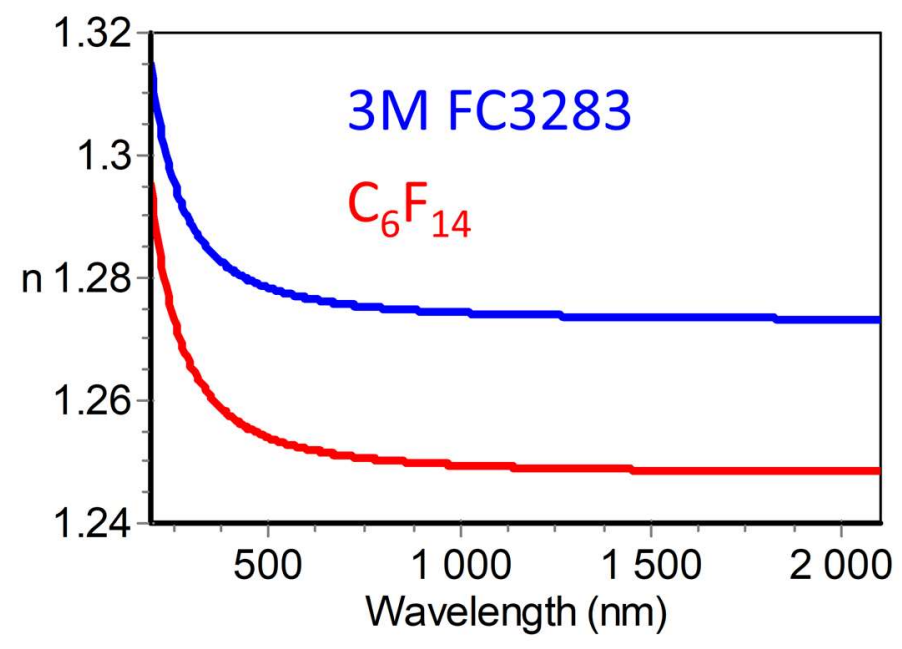

Figure 7 Real part of refractive index for tetradecafluorohexane in red and commercial $3 M^{\mathrm{TM}}$ FC3283 in blue

4.3. Application for concentration estimation of ovalbumine solution 
The method was applied in the biology field to evaluate protein concentration in solutions. We synthesized several bovine serum albumin (BSA) aqueous solutions and estimated their concentration with a Thermo Fischer nanodrop UV-Visible spectrophotometer. This device specifications are $2 \%$ accuracy and $0.1 \mathrm{mg} / \mathrm{ml}$ reproducibility. The ellipsometric characterization was done in parallel. Optical properties are described by a single TaucLorentz dispersion formula over the range $4 \mathrm{eV}$ to $6.5 \mathrm{eV}$. It is noticeable that both methods consume very little material, typically one drop or $50 \mu \mathrm{l}$. Figure $9 \mathrm{a}$ summarizes the ellipsometric determination of the extinction coefficient $k$ and finally illustrates the linear dependence between the different concentrations and the value of the extinction coefficient at $200 \mathrm{~nm}(6.2 \mathrm{eV})$ deduced from ellipsometry analysis.
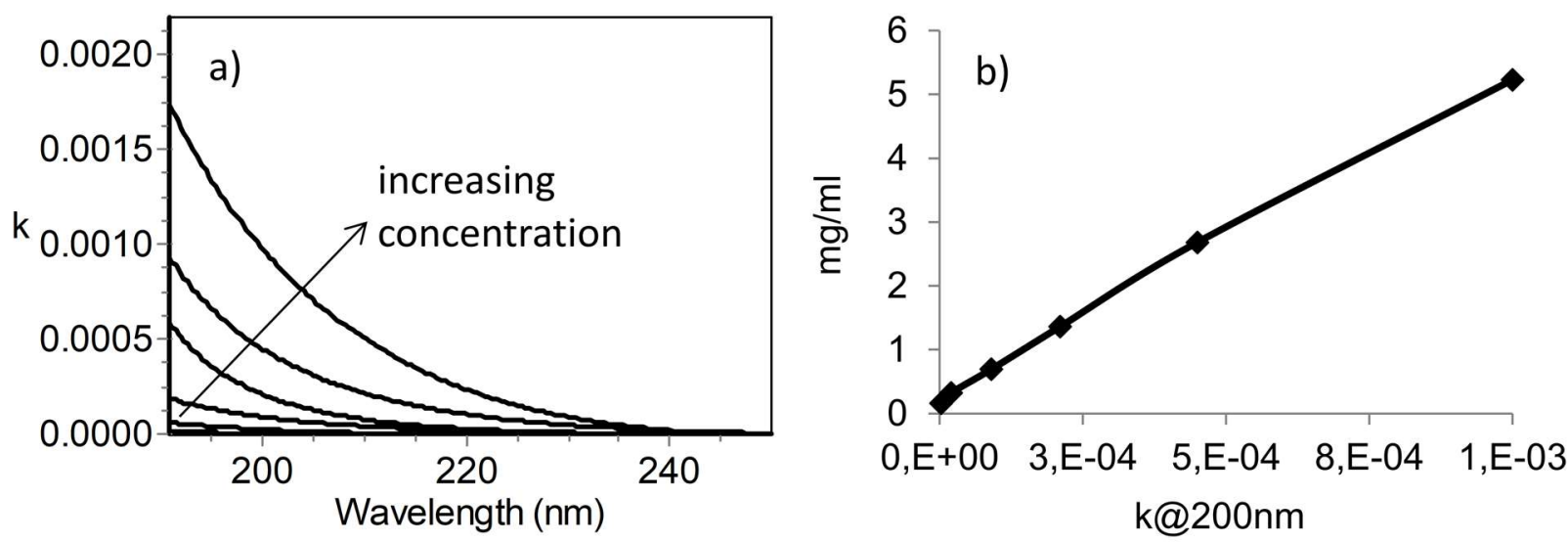

Figure 8 Experiments on BSA solutions. a) Extinction coefficient $k$ obtained by ellipsometry. b) Dependence of concentration with $k$ at $200 \mathrm{~nm}$

\section{Conclusions}

We have demonstrated the possibility to characterize optical properties of bulk liquids with a standard ellipsometer using a dedicated accessory. This accessory prevents the waves at the liquid surface and regarding modeling method, exploits the summation of multiple incoherent reflected beams. It allows the characterization of the optical properties of any variety of liquids with accuracy in the range of $0.1 \%$. This new method shows comparable results with reference techniques like spectrophotometry but presents the advantage to extract simultaneously real and imaginary part of the optical index thanks to the standard modeling package available with the instrument. The method has been used for 
characterization of pure liquids for validation purposes and then it has been applied to several solutions where it shows no disadvantages even in the case of absorbing liquids. Future application of the method will include studies of solutions of nanoparticle in a short term then its extension to the infrared spectral range.

\section{Acknowledgement}

The authors are grateful to Michelle Sestak from Horiba Instruments Inc for her comments and revision of the manuscript.

\section{References}

[1] H.G. Tompkins, E.A.Irene, Handbook of Ellipsometry, William Andrews, New York (2005)

[2] Kurt Hingerl, Polarimetric and other optical probes for the Solid-Liquid interface, Ellipsometry at the nanoscale, Springer, 493 (2013)

[3] J. P. R. Day, C. D. Bain, Ellipsometric Study of Depletion at Oil-Water Interfaces, PHYS. REV. E, 76, 041601 (2007)

[4] R. A. Synowicki, Greg K. Pribil, Gerry Cooney, Craig M. Herzinger, Steven E. Green, Roger H. French, Min K.Yang, John H. Burnett and Simon Kaplan, Fluid refractive index measurements using rough surface and prism minimum deviation techniques, JOURNAL OF VACUUM SCIENCE \& TECHNOLOGY B, 22, 3450 (2004)

[5] R. Greef, J.G. Frey, The water-like film on water,. PHYS. STAT. SOL. C, 5, 1184, (2008)

[6] K.Y. Bang, S. Lee, H. Oh,I. An and H. Lee*, Determination of the Optical Functions of Various Liquids by Rotating Compensator Multichannel Spectroscopic Ellipsometry, BULL. KOREAN CHEM. SOC., 26, 6, 947 (2005) 
[7] M.Kildemo, R.Ossikovski and M.Stchakovsky, Measurement of the absorption edge of thick transparent substrates using the incoherent reflection model and spectroscopic UVvisible-near IR ellipsometry, THIN SOLID FILMS, 313-314, 108, (1998)

[8] http://iapws.org/relguide/rindex.pdf

[9] M Daimon and A. Masumura, Measurement of the refractive index of distilled water from the near-infrared region to the ultraviolet region, APPLIED OPTICS, 46,18, 3811, (2007)

[10] S. Kedenburg, M. Vieweg, T. Gissibl, and H. Giessen, Linear refractive index and absorption measurements of nonlinear optical liquids in the visible and near-infrared spectral region, OPTICAL MATERIALS EXPRESS, 2, 11, 1588, (2012)

[11] E.D.Palik, Handbook of optical constants of solids, Academic Press, Inc (1985)

[12] L.Kou, D.Labrie, P.Chylek, Refractive indices of water and ice in the 0.65 to $2.65 \mu \mathrm{m}$ spectral range, APPLIED OPTICS, 32, 19, 3531 (1993)

[13] G. E. Jellison Jr. and F. A. Modine, Parameterization of the optical functions of amorphous materials in the interband region, APPLIED PHYSICS LETTERS, 69, 3, 371, (1996). 